\title{
Extractos de plantas del semidesierto en la inducción del crecimiento de tomate (Lycopersicon esculentum Mill)
}

\section{Extracts of plants from the semidesert in the induction of tomato (Lycopersicon esculentum Mill) growth}

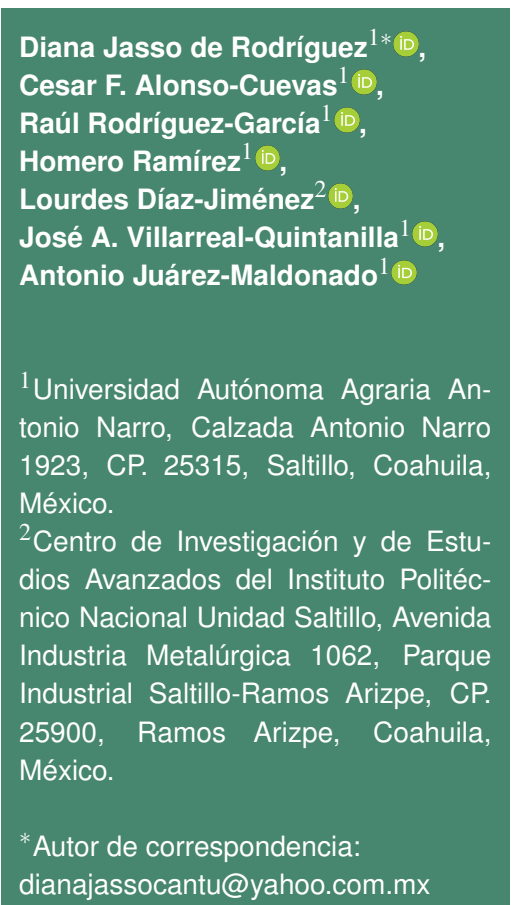

Artículo científico

Recibido: 13 de junio de 2019

Aceptado: 21 de enero de 2020

Como citar: Jasso de Rodríguez D, Alonso-Cuevas CF, RodríguezGarcía R, Ramírez H, Díaz-Jiménez L, Villarreal-Quintanilla JA, JuárezMaldonado A (2020) Extractos de plantas del semidesierto en la inducción del crecimiento de tomate (Lycopersicon esculentum Mill). Ecosistemas y Recursos Agropecuarios 7(1): e2342. DOI: 10.19136/era.a7n1.2342

RESUMEN. En las zonas semiáridas del noreste de México, se han identificado especies con alto contenido de polifenoles y actividad antioxidante, que pueden ser utilizadas como promotores de crecimiento y calidad del fruto en plantas de tomate. El objetivo fue conocer el efecto de extractos de Rhus trilobata, Rhus muelleri, Flourensia microphylla, Flourensia retinophylla y Cucurbita foetidissima, como promotores de crecimiento y calidad del fruto en plantas de tomate saladette, al compararlos con los biorreguladores AIA, AG y 6-BAP. La dosis del extracto y biorreguladores fue de $75 \mathrm{mg} \mathrm{L}^{-1}$, al trasplante, y a los 34 y 55 días después del trasplante. Cada quince días se evaluó el crecimiento longitudinal y diámetro de tallo, número de hojas, número de frutos, peso seco, peso y producción de fruto, además de las variables de calidad: diámetro polar y ecuatorial, firmeza, $\mathrm{pH}$, sólidos solubles totales, contenido de licopeno y vitamina C. El experimento se estableció bajo un diseño completamente al azar con nueve tratamientos y 12 repeticiones. En general los extractos incrementaron la longitud y diámetro de tallo, peso seco de hojas, número y peso de frutos, y producción de fruto, con resultados similares al biorregulador 6-BAP. El extracto de $R$. muelleri mostró la mayor efectividad en promover el crecimiento y producción de fruto de tomate por lo que representa una alternativa para la formulación de un bioestimulante de origen biológico que mejore la productividad de las plantas de tomate.

Palabras clave: Biorreguladores, extractos de plantas, Lycopersicon esculentum Mill., producción de fruto, promotor de crecimiento.

ABSTRACT. In the semi-arid areas of northeastern Mexico, species with high polyphenol content and antioxidant activity have been identified, which can be used as growth and fruit quality promoters in tomato plants. The objective was to know the effect of Rhus trilobata, Rhus muelleri, Flourensia microphylla, Flourensia retinophylla and Cucurbita foetidissima extracts, as growth and fruit quality promoters in saladette tomato plants, when compared with bioregulators, AIA, AG and 6-BAP. The dose of the extract and bioregulator was $75 \mathrm{mg} \mathrm{L}^{-1}$, at the transplant and at 34 and 55 days after the transplant. Every fifteen days the longitudinal growth and stem diameter, number of leaves, number of fruits, dry weight, in addition to weight and fruit production were evaluated, in addition to the quality variables: polar and equatorial diameter, firmness, $\mathrm{pH}$, soluble solids total, lycopene and vitamin $\mathrm{C}$ content. The experiment was established under a completely randomized design with nine treatments and 12 repetitions. In general, extracts increased stem length and diameter, dry leaf weight, number and weight of fruits, and fruit production, with results similar to the 6-BAP bioregulator. The extract of $R$. muelleri showed the greatest effectiveness in promoting the growth and production of tomato fruit, which is why it represents an alternative for the formulation of a bio-stimulant of biological origin that improves the productivity of tomato plants.

Key words: Bioregulators, extracts plants, Lycopersicon esculentum Mill., fruit yield, growth promoter. 


\section{INTRODUCCIÓN}

México ocupa el décimo lugar a nivel mundial en la producción de tomate (Lycopersicon esculentum Mill) y el primero en exportación, además de que es la hortaliza de mayor importancia por su producción en el país (FIRA 2017). Por su importancia económica su cultivo exige una continua búsqueda de tecnologías que contribuyan a mejorar el rendimiento y calidad del fruto. En el contexto de una agricultura sostenible, se utilizan técnicas de manejo del cultivo con sustancias de origen natural (bioestimulantes), que tienen efectos benéficos sobre el crecimiento y desarrollo de las plantas, resistencia al estrés, rendimiento y calidad de los cultivos (Parađiković et al. 2018).

Investigaciones con extractos polifenólicos de la corteza de abeto (Picea abies) reportan la mejora de la capacidad de germinación de semillas de Glycine max L. y Helianthus annuus (Tanase et al. 2011), en Ocimum basilicum L. se incrementa la elongación de tejidos y la acumulación de biomasa (Talmaciu et al. 2015). Por otro lado, trabajos realizados con extractos polifenólicos de semilla de uva estimulan el alargamiento de la raíz de avena (Avena sativa) y maíz (Zea mays), además de la acumulación de biomasa (Ingat et al. 2011). También se tienen reportes que los extractos polifenólicos de otras especies, tienen actividad antioxidante y capacidad antifúngica (Jasso de Rodríguez et al. 2015).

En las zonas semiáridas del noreste de México se han identificado especies con alto contenido de polifenoles y actividad antioxidante. Por ejemplo, en el extracto de etanol de las hojas de Rhus muelleri se han identificado compuestos de naturaleza alcohólica, esterol, fenólica, flour y lupene, con contenido total de fenoles de $37.9 \mathrm{mg} 100 \mathrm{mg}^{-1}$ Equivalentes de Ácido Gálico y actividad antioxidante del 73.4\% (Jasso de Rodríguez et al. 2015). Por otra parte, en el extracto acuoso de tallos de $R$. trilobata, se identificaron compuestos flavonoides, ácidos fenólicos, ácidos grasosos y compuestos glicosilados con contenido de $9.41 \mathrm{mg} 100 \mathrm{mg}^{-1}$ Equivalentes de ácido Gálico y $86.0 \%$ de actividad antioxidante (VarelaRodríguez et al. 2019). También se reporta que extractos de etanol de las hojas de Flourensia microphylla y $F$. retinophylla contienen un CTF de 31.4 y $32.5 \mathrm{mg} 100^{-1} \mathrm{mg}$ Equivalentes de Ácido Gálico, y actividad antioxidante de 94.9 y $50.0 \%$, respectivamente. También para el extracto de F. microphylla se identificaron compuestos de naturaleza amino fenólico, éster de ácido graso y poliol. Mientras que en el extracto de $F$. retinophylla se identificaron compuestos de naturaleza amida (Jasso de Rodríguez et al. 2017). Por otro lado, trabajos realizados con semillas de Cucurbita foetidissima, reportan altos contenidos de ácidos grasos insaturados que tienen alta estabilidad oxidativa y elevado porcentaje de insaturación total (Stevenson et al. 2007). Por lo anterior, el objetivo del presente trabajo fue conocer el efecto de cinco extractos de plantas del semidesierto como promotores de crecimiento y calidad del fruto en plantas de tomate saladette, al compararlos con los biorreguladores, AIA, AG y 6-BAP.

\section{MATERIALES Y MÉTODOS}

\section{Ubicación y establecimiento del experimento}

El estudio se realizó en la Universidad Autónoma Agraria Antonio Narro, en Saltillo, Coahuila, México, durante el otoño-invierno 20172018, en un invernadero del Departamento de Forestal, con estructura metálica, cubierta de plástico blanco (calibre 720) y placas de policarbonato en las paredes laterales.

Se utilizó semillas de tomate saladette híbrido EL CID F1, que se sembró en charolas de poliestireno de 200 cavidades. Cuando las plántulas tenían en promedio $14.2 \mathrm{~cm}$ de altura a los 34 días después de la siembra (DDS), se trasplantaron a macetas de plástico de $20 \mathrm{~L}$, que tenían como sustrato $15 \mathrm{~L}$ de peat moss y perlita (1:1). Las macetas se distribuyeron a distancia de $50 \mathrm{~cm}$ entre plantas y 75 $\mathrm{cm}$ entre filas.

El experimento se estableció bajo un diseño completamente al azar, con nueve tratamientos, cinco extractos de plantas: Rhus trilobata (RT), R. muelleri $(\mathrm{RM})$, Flourensia microphylla (FM), F. retinophylla (FR) y Cucurbita foetidissima (CF); tres biorreguladores: Ácido indolacético (AIA), Ácido giberélico 
(AG) y 6 Bencil aminopurina (6-BAP); y un control (CTRL) sin extracto ni biorregulador, con 12 repeticiones por tratamiento con una planta por repetición. En RT, RM, FM, y FR los extractos fueron de las hojas y en CF fue del fruto. Los extractos de las plantas se obtuvieron siguiendo la técnica reportada por Ramírez et al. (2001). De la muestra seca y molida de las hojas y fruto se tomaron $10 \mathrm{~g}$ que se colocaron en un matraz erlenmeyer, al que se le agregaron $500 \mathrm{~mL}$ de metanol al $80 \%$ y se mantuvieron por $24 \mathrm{~h}$ en congelación a $-15^{\circ} \mathrm{C}$, para luego filtrar el extracto usando papel Whatman \# 1, al filtrado se le agregaron $500 \mathrm{~mL}$ de metanol al $100 \%$ y se colocó durante $4 \mathrm{~h}$ en congelación a $-15{ }^{\circ} \mathrm{C}$, para filtrar de nuevo. Luego de repetir el proceso, se mezclaron los filtrados para luego evaporar en un rotavapor (Yamato Scientific Co.,Ltd., Tokyo, Japan), a $50{ }^{\circ} \mathrm{C}$. La purificación de la muestra se realizó mediante una cápsula de silica gel Sep Pack C18 para obtener el extracto final que se utilizó en el experimento.

La aplicación de la concentración de los extractos y los biorreguladores fue de $75 \mathrm{mg} \mathrm{L}^{-1}$, los cuales se combinaron con $1.5 \mathrm{~mL} \mathrm{~L}^{-1}$ del adherente Bionex. Los extractos y biorreguladores se asperjaron a punto de goteo: la primera aplicación se realizó al trasplante (34 DDS), la segunda a los 34 días después de trasplante (DDT), cuando las plantas presentaban un $50 \%$ de floración y la tercera a los 55 DDT, cuando se presentaron los primeros frutos. El cultivo se manejó a un tallo durante el ciclo del cultivo, con el fin de detener el crecimiento longitudinal, se realizó un corte apical a los 101 DDT, cuando la mayoría de las plantas contaban con 6 racimos.

\section{Evaluación de variables de crecimiento de la planta}

Las variables de crecimiento evaluadas fueron: crecimiento longitudinal de tallo con una cinta métrica $(\mathrm{cm})$, crecimiento de diámetro de tallo con un vernier digital $(\mathrm{mm})$, número de hojas y número de frutos. Los datos se obtuvieron cada 15 días a partir del trasplante. La cosecha de frutos se realizó a los 126 y 157 DDT. Los frutos se cosecharon a medida que maduraron, del primero al sexto racimo. Para determinar el peso de fruto y producción, solo se consideraron los frutos con peso mayor a $50 \mathrm{~g}$. Al final del ciclo (157 DDT), se cosechó la parte aérea (hojas y tallo) de cada planta y se determinó el peso seco de cada parte y el total. Para determinar la longitud y el peso seco de la raíz de las plantas, se eliminó con agua el sustrato, con la finalidad de separar las raíces del sustrato.

\section{Evaluación de calidad del fruto}

Las pruebas de calidad de fruto se efectuaron en cinco tomates del primer racimo, debido a que presentaron uniformidad de maduración en todos los tratamientos, cuando estaban en un grado de maduración cinco (Casierra-Posada y Aguilar-Avendaño 2008). Las variables evaluadas fueron: diámetro polar, diámetro ecuatorial, firmeza, $\mathrm{pH}$, sólidos solubles totales (SST), contenido de licopeno y contenido de vitamina C.

Las mediciones de firmeza se realizaron en el eje ecuatorial en dos puntos opuestos en cada uno de los cinco frutos, con un penetrómetro (QA supplies, Norfolk, VA, USA), las mediciones se expresaron en kilogramo por centímetro cuadrado (Kgf $\mathrm{cm}^{-2}$ ) (Pérez-Labrada et al. 2014). El pH se midió en la pulpa molida del tomate, con un potenciómetro marca Hanna (Instruments Inc., Romania), para lo cual se utilizó solución buffer para calibrar el equipo (AOAC 1990). Los sólidos solubles totales se midieron con un refractómetro (ATAGO, Co. Ltd., Japan), de acuerdo al método 932.12 de la Association of Official Analytical Chemists (AOAC 1990). La cuantificación del contenido de licopeno y vitamina C se realizó en tomate molido en un cromatógrafo de líquidos (HPLC), siguiendo las metodologías reportadas por Arias et al. (2000) y Gutiérrez et al. (2007), respectivamente.

\section{Análisis estadístico de datos}

Los datos se analizaron con el programa de análisis estadístico SAS 9.0, para determinar diferencias entre medias se realizó la prueba de Tukey a una probabilidad del $0.05 \%$. 


\section{RESULTADOS}

\section{Crecimiento longitudinal y diámetro de tallo}

Para la variable crecimiento longitudinal de tallo (Figura 1), se detectaron diferencias entre tratamientos ( $p \leq 0.05$ ), el biorregulador AG produjo el mayor crecimiento de tallo durante el ciclo de cultivo, seguido en crecimiento por los cinco extractos vegetales (RT, RM, FM, FR y CF) y los dos biorreguladores (AIA y 6-BAP), que fueron estadísticamente iguales, presentando la menor longitud el tratamiento control. Por otra parte, el crecimiento del diámetro de tallo de las plantas de tomate aumentó de forma significativa desde el inicio hasta los 30 DDT (Figura 2), a partir de esta fecha y hasta los 45 DDT el crecimiento disminuyó en los tratamientos. Los mayores diámetros de tallo fueron para los cinco extractos de plantas, el biorregulador 6-BAP y el control fueron estadísticamente iguales $(p=0.05)$, mientras que el tratamiento AG presentó el menor diámetro de tallo.

\section{Crecimiento aéreo y radicular de la planta}

Se tuvieron diferencias significativas $(p \leq 0.05)$ entre tratamientos para las variables: número de hojas, peso seco de hojas, tallo y peso total aéreo de la planta; además de longitud y peso seco de raíz (Tabla 1). El tratamiento AG, presentó el mayor número de hojas lo cual se atribuye al mayor crecimiento longitudinal de tallo que tuvieron las plantas durante el ciclo del cultivo, en comparación con los otros tratamientos que tuvieron menor número y fueron estadísticamente iguales. Para el peso seco de hojas y aéreo total, los extractos de las cinco especies evaluadas tuvieron un peso similar al de las plantas a las que se les aplicó el tratamiento AG. En lo que respecta, al peso de tallo, los extractos de RT y RM promovieron pesos similares al tratamiento con AG. En relación a la longitud de raíz el extracto de FM, AIA y 6-BAP presentaron la mayor longitud y fueron estadísticamente iguales, mientras que los tratamientos restantes tuvieron menor longitud y fueron iguales estadísticamente. Para el peso seco de raíz, los extractos de RT, RM y FM presentaron un efecto similar al tratamiento 6-BAP.

\section{Variables de rendimiento}

Los análisis estadísticos de las variables de rendimiento presentaron diferencias significativas ( $p$ $\leq 0.05$ ) entre tratamientos (Tabla 2). El número de frutos de los extractos de RT, RM, FM y CF presentó un efecto similar al del tratamiento 6-BAP. El peso promedio de fruto por planta de los extractos RT, RM y FM fue similar al que tuvieron las plantas con el tratamiento 6-BAP con valores de 94.8 a $96.1 \mathrm{~g}$. El extracto de RM fue el que sobresalió en producción con $3.1 \mathrm{~kg} \mathrm{planta}^{-1}$, junto con el biorregulador 6-BAP con $2.9 \mathrm{~kg} \mathrm{planta}^{-1}$. Los resultados muestran que el extracto de RM, presentó la mayor efectividad como estimulante en las tres variables de rendimiento en las plantas de tomate.

\section{Variables de calidad de fruto}

Para las variables diámetro polar y ecuatorial y contenido de vitamina $\mathrm{C}$ se tuvieron diferencias significativas ( $p \leq 0.05)$, entre tratamientos, mientras que para las variables firmeza, $\mathrm{pH}$, SST y contenido de licopeno no se encontraron diferencias significativas. Para diámetro polar los extractos de RT, FM y FR fueron estadísticamente similares al 6-BAP presentando los valores más altos de diámetro. En tanto que, para el diámetro ecuatorial, los cinco extractos fueron similares al 6-BAP, con diámetros entre 50.7 y $51.4 \mathrm{~mm}$ (Tabla 2). En relación con el contenido de vitamina $\mathrm{C}$, los extractos de RT y RM tuvieron contenidos de vitamina $\mathrm{C}$ de 10.54 y $11.18 \%$, respectivamente, valores que son iguales estadísticamente al que se tuvo con el biorregulador AG (10.22\%), valores que son estadísticamente superiores a los que se tuvieron con los extractos FM, FR, CF y los biorreguladores AIA y 6-BAP (Figura 3).

\section{DISCUSIÓN}

\section{Crecimiento longitudinal y de diámetro del tallo.}

Los extractos vegetales, contienen compuestos bioactivos que incrementan el crecimiento en longitud y diámetro en las plantas (Figuras 1 y 2). Se ha demostrado que lo extractos fenólicos de algunas especies de plantas, actúan como bioestimulantes de procesos fisiológicos, como la elongación radicular de 


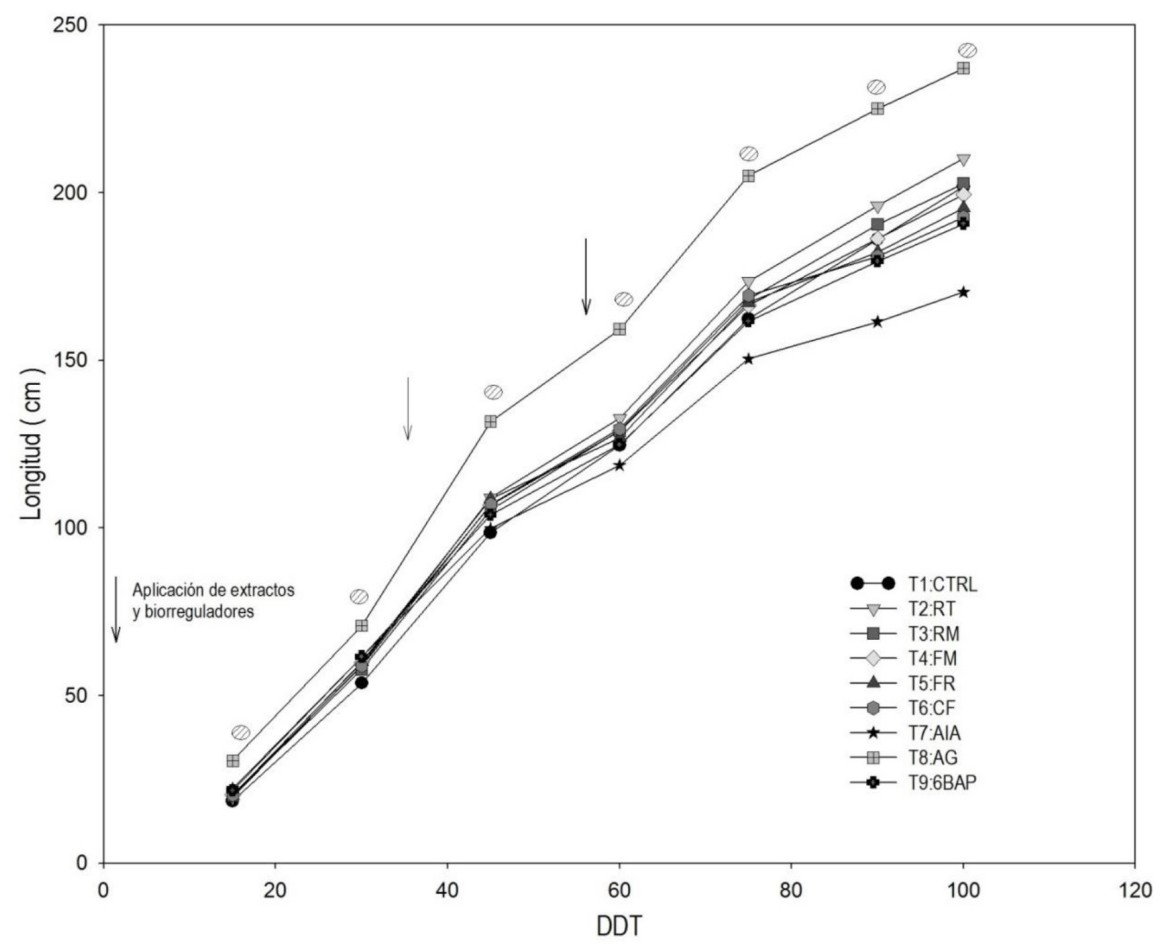

Figura 1. Crecimiento longitudinal de tallo. Cada punto representa la media de 12 repeticiones; Representa diferencia estadística significativa ( $p \leq 0.05)$.

las plántulas de avena y maíz, así como la acumulación de biomasa verde (Ignat et al. 2011). Sobre lo mismo Tanase et al. (2013), encontraron que el extracto acuoso de la corteza de Picea abies, influyó en el crecimiento de los explantes de Lavandula angustifolia Mill, aumentando el crecimiento longitudinal del tallo principal, número de hojas formadas y síntesis de pigmentos foto asimilados. Por lo que el efecto de inducción de crecimiento longitudinal y diámetro del tallo en los tratamientos de los extractos evaluados, podría atribuirse a los compuestos fenólicos que contienen los extractos vegetales evaluados (Jasso de Rodríguez et al. 2015, Jasso de Rodríguez et al. 2017).

El mayor crecimiento longitudinal del tallo observado en el tratamiento AG (Figura 1), se puede deber a una mayor estimulación de la división y elongación celular en la porción subapical de los tallos y también en el meristemo intercalar, como ha sido reportado (Ramírez et al. 2005), por otra parte al promover mayor crecimiento longitudinal se redujo el crecimiento del diámetro de tallo (Figura 2). Los extractos y biorreguladores de crecimiento AIA y 6-BAP promovieron la división y elongación celular, que estimuló el crecimiento longitudinal y diámetro de tallo, lo que ha sido comprobado para los biorreguladores por Giovannoni (2001) y Gravel et al. (2007). Para los extractos, se ha demostrado que los compuestos polifenólicos de corteza de Picea abies L, provocan mayor división celular (índice mitótico) y crecimiento de tallo en plántulas de Ocimum basilicum L. (Tanase et al. 2018).

\section{Crecimiento aéreo y radicular de la planta}

Los compuestos fenólicos de las plantas pueden actuar de forma similar a las hormonas de crecimiento, auxinas y citocininas, promoviendo el crecimiento foliar y biomasa (Ignat et al. 2013). En la presente investigación los extractos de las plantas promovieron mayor número de hojas, peso seco de hojas, tallo y biomasa aérea total, así como longitud y peso seco de raíz que fue similar estadísti- 


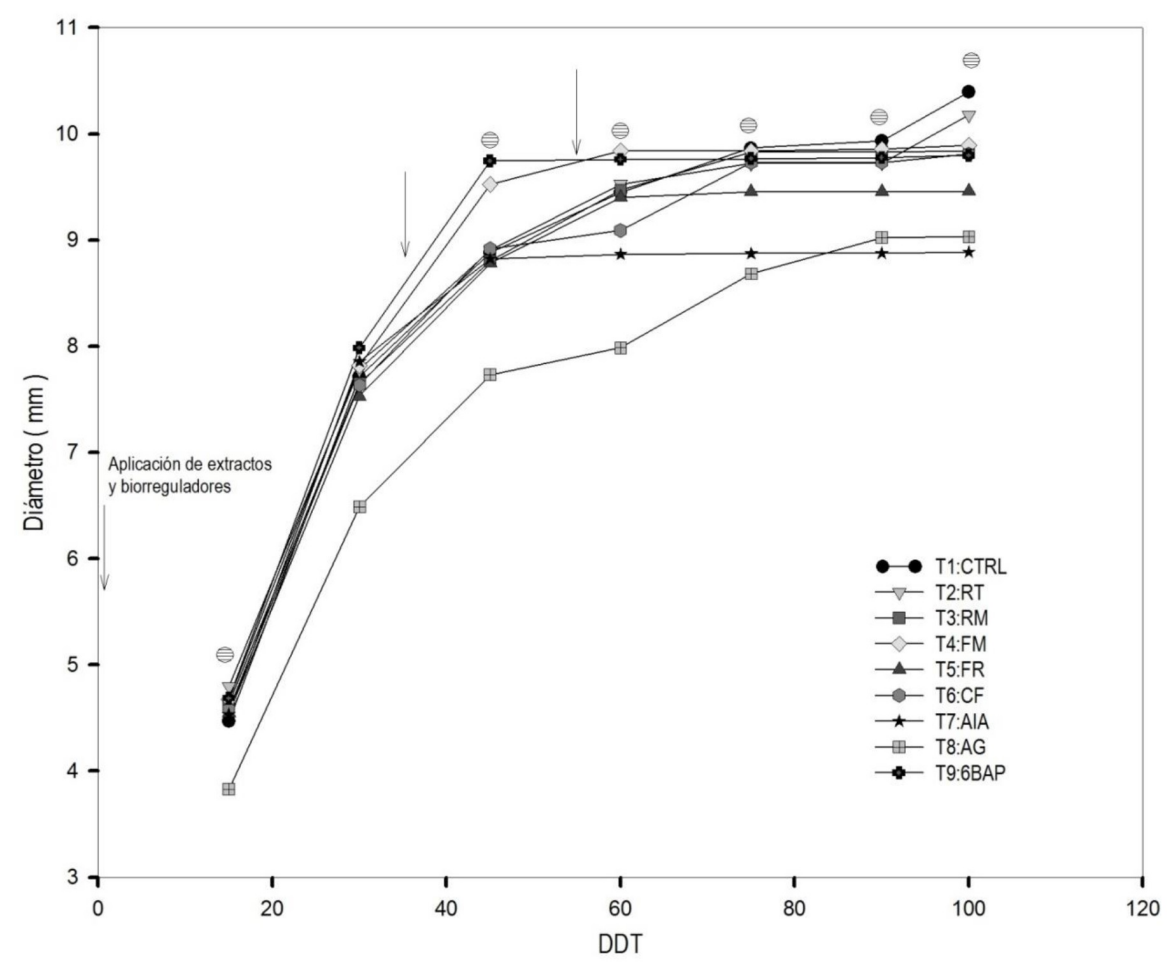

Figura 2. Crecimiento de diámetro de tallo. Cada punto representa la media de 12 repeticiones; $\theta$ Representa diferencia estadística significativa $(p \leq 0.05)$.

Tabla 1. Efecto de los tratamientos en variables agronómicas.

\begin{tabular}{ccccccc}
\hline Tratamiento & No. de hojas & $\begin{array}{c}\text { PS Hojas } \\
(\mathrm{g})\end{array}$ & $\begin{array}{c}\text { PS Tallo } \\
(\mathrm{g})\end{array}$ & $\begin{array}{c}\text { Peso seco aéreo total } \\
(\mathrm{g})\end{array}$ & $\begin{array}{c}\text { Long. de raíz } \\
(\mathrm{cm})\end{array}$ & $\begin{array}{c}\text { Peso seco raíz } \\
(\mathrm{g})\end{array}$ \\
\hline CTRL & $24.1^{b}$ & $129.6^{a}$ & $44.3^{a}$ & $174^{a}$ & $75.3^{a b}$ & $8.6^{a}$ \\
RT & $24.3^{b}$ & $102.3^{b c}$ & $36.8^{a b c}$ & $139.1^{b}$ & $57.4^{b}$ & $5.3^{b}$ \\
RM & $23.5^{b}$ & $99.8^{b c}$ & $35.8^{a b c}$ & $135.6^{b c}$ & $79^{a b}$ & $4.3^{b c}$ \\
FM & $24.0^{b}$ & $90.5^{b c}$ & $33^{b c d}$ & $123.6^{b c}$ & $87.9^{a}$ & $4.2^{b c}$ \\
FR & $24.5^{b}$ & $92.9^{b c}$ & $30.5^{c d}$ & $121.5^{b c}$ & $77.7^{a b}$ & $3.0^{d}$ \\
CF & $23.4^{b}$ & $89.2^{b c}$ & $29.4^{c d}$ & $118.6^{b c}$ & $82.1^{a b}$ & $3.8^{c d}$ \\
AIA & $23.4^{b}$ & $79.6^{c}$ & $25.4^{d}$ & $105^{c}$ & $98^{a}$ & $3.5^{c d}$ \\
AG & $28.6^{a}$ & $92.9^{b c}$ & $41.5^{a b}$ & $134.4^{b c}$ & $77.8^{a b}$ & $4.1^{b c d}$ \\
6-BAP & $21.6^{b}$ & $104.6^{b}$ & $30.7^{c d}$ & $135.4^{b c}$ & $90.2^{a}$ & $4.5^{b c}$ \\
C.V. & 11.31 & 18.90 & 21.18 & 18.78 & 24.47 & 20.58 \\
\hline Valores con la misma letra en columnas son estadísticamente iguales de acuerdo con la prueba de Tukey $(\mathrm{p} \leq 0.05)$.
\end{tabular}

camente, a la que se tuvo con los biorreguladores. El comportamiento de los extractos evaluados es relevante, porque están conformados químicamente por una matriz de compuestos que podrían estar actuando en sinergia para promover la estimulación en las plantas (Jasso de Rodríguez et al. 2015), mientras que los biorreguladores son compuestos puros. En relación con lo anterior, los extractos fenólicos a bajas concentraciones de Asclepias syriaca, semilla de Vitis vinífera, y corteza de Picea abies estimu- laron la elongación de la radícula en semillas de Phaseolus vulgaris (Ignat et al. 2009). Mientras que el extracto polifenólico de semilla de Vitis vinífera estimuló el alargamiento de la raíz de avena (Avena sativa) y maíz (Zea mays), así como la acumulación de biomasa (Ingat et al. 2011). Lo que confirma los resultados del crecimiento de la raíz obtenidos por efecto de los extractos de las especies evaluadas en el presente estudio. 
Tabla 2. Efecto de los tratamientos en el rendimiento de cultivo.

\begin{tabular}{cccc}
\hline Tratamiento & Número de frutos & $\begin{array}{c}\text { Peso medio de fruto por planta } \\
(\mathrm{g})\end{array}$ & $\begin{array}{c}\text { Producción } \\
\left.(\mathrm{kg} \mathrm{planta})^{-1}\right)\end{array}$ \\
\hline CTRL & $34^{a}$ & $105^{a}$ & $3.5^{a}$ \\
RT & $28.6^{a b}$ & $96.1^{a b}$ & $2.7^{b c}$ \\
RM & $32^{a b}$ & $95.9^{a b}$ & $3.0^{a b}$ \\
FM & $28^{a b}$ & $94.8^{a b}$ & $2.6^{b c}$ \\
FR & $27^{b}$ & $91.8^{b}$ & $2.4^{b c}$ \\
CF & $30.2^{a b}$ & $87.7^{b c}$ & $2.6^{b c}$ \\
AIA & $25.9^{b c}$ & $87.9^{b c}$ & $2.3^{c d}$ \\
AG & $20.3^{c}$ & $79.1^{c}$ & $1.6^{d}$ \\
6-BAP & $31.4^{a b}$ & $95.1^{a b}$ & $2.9^{a b c}$ \\
C.V. & 18.04 & 10.16 & 20.08 \\
\hline
\end{tabular}

Valores con la misma letra en columnas son estadísticamente iguales de acuerdo con la prueba de Tukey $(p \leq 0.05)$.

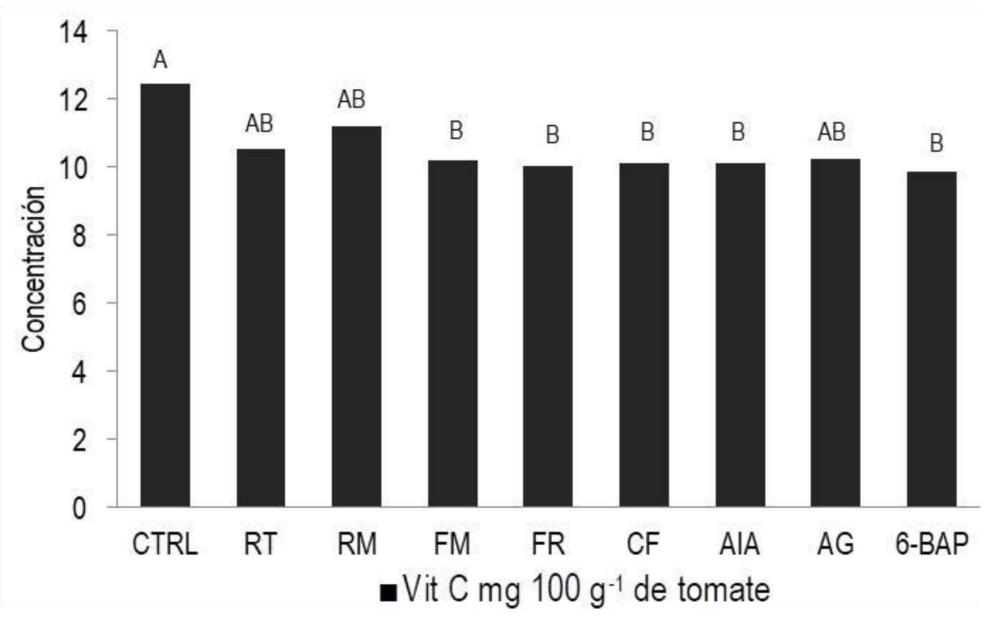

Figura 3. Contenido promedio de vitamina $\mathrm{C}$ por tratamiento; barras con la misma letra son estadísticamente iguales de acuerdo con la prueba de Tukey $(p \leq 0.05)$.

\section{Variables de rendimiento}

El extracto de R. muelleri, tuvo mayor efectividad al estimular mayor peso, producción y número de frutos de tomate, con valores similares estadísticamente a los obtenidos con el biorregulador 6-BAP (Tabla 2). La efectividad del extracto de R. muelleri para incrementar el rendimiento del tomate, podría atribuirse al alto contenido de fenoles y compuestos de naturaleza, esterol, fenólica, alcohólica, flour y lupene, y a la interacción sinergística entre los componentes del extracto (Jasso de Rodríguez et al. 2015). Al respecto, Paradikovic et al. (2018), reportan que la utilización del bioestimulante Radifarm, que contiene compuestos naturales de plantas como aminoácidos, glicósidos, saponinas, polisacáridos y otros compuestos, mejora las variables de rendimiento de fruto en Capsicum annuum, debido a la sinergia de los compuestos del bioestimulante.

\section{Variables de calidad}

La norma de calidad del tomate NMX-FF-0311997 (SCFI 1988), establece que los frutos con un diámetro ecuatorial entre 51 y $61 \mathrm{~mm}$ son clasificados como de calidad mediana, por lo que los frutos cosechados en el experimento corresponden a esta calidad. La firmeza de los frutos tuvo valores entre 4.0 y $4.6 \mathrm{Kgf} \mathrm{cm}^{-2}$. Al respecto Ramírez et al. (2016), en un estudio realizado sobre el efecto de biorreguladores en tomate, reportó valores de firmeza de frutos de 3.5 a $4.5 \mathrm{Kgf} \mathrm{cm}^{-2}$, señalando que la presencia de giberelinas durante la maduración del fruto promueven la rigidez en la piel y las membranas externas. En el presente estudio, los valores de firmeza reportados indican que los extractos aplicados es- 
Tabla 3. Efecto de los tratamientos en la calidad de frutos

\begin{tabular}{lcccccc}
\hline Tratamiento & $\begin{array}{c}\text { Diámetro polar } \\
(\mathrm{mm})\end{array}$ & $\begin{array}{c}\text { Diámetro ecuatorial } \\
(\mathrm{mm})\end{array}$ & $\begin{array}{c}\text { Firmeza } \\
\mathrm{Kgf} \mathrm{cm}{ }^{-2}\end{array}$ & $\mathrm{pH}$ & $\begin{array}{c}\mathrm{SST} \\
\left({ }^{\circ} \mathrm{Brix}\right)\end{array}$ & $\begin{array}{c}\text { Licopeno } \\
\left(\mathrm{mg} 100 \mathrm{~g}^{-1}\right)\end{array}$ \\
\hline $\mathrm{CTRL}$ & $63.6^{a}$ & $52.2^{a}$ & $4.6^{a}$ & $4.5^{a}$ & $4.5^{a}$ & $18.4^{a}$ \\
$\mathrm{RT}$ & $60.3^{a b}$ & $50.9^{a}$ & $4.4^{a}$ & $4.6^{a}$ & $4.7^{a}$ & $19.2^{a}$ \\
$\mathrm{RM}$ & $59.1^{b}$ & $50.8^{a}$ & $4.4^{a}$ & $4.6^{a}$ & $4.5^{a}$ & $20.3^{a}$ \\
$\mathrm{FM}$ & $59.2^{a b}$ & $51.1^{a}$ & $4.4^{a}$ & $4.7^{a}$ & $4.5^{a}$ & $18.5^{a}$ \\
$\mathrm{FR}$ & $59.6^{a b}$ & $50.7^{a}$ & $4.3^{a}$ & $4.5^{a}$ & $4.5^{a}$ & $20.8^{a}$ \\
$\mathrm{CF}$ & $57.9^{b}$ & $50.4^{a}$ & $4.0^{a}$ & $4.6^{a}$ & $4.4^{a}$ & $18.9^{a}$ \\
$\mathrm{AIA}$ & $55.8^{b}$ & $49.1^{a b}$ & $4.0^{a}$ & $4.5^{a}$ & $4.5^{a}$ & $20.1^{a}$ \\
$\mathrm{AG}$ & $57.6^{b}$ & $46^{b}$ & $4.1^{a}$ & $4.6^{a}$ & $4.5^{a}$ & $21^{a}$ \\
6-BAP & $59.4^{a b}$ & $51.4^{a}$ & $4.5^{a}$ & $4.6^{a}$ & $4.4^{a}$ & $20.6^{a}$ \\
C.V. & 5.83 & 5.03 & 46.82 & 7.33 & 7.06 & 37.96 \\
\hline Valores con la misma letra en columnas son estadísticamente iguales de acuerdo con la prueba de Tukey \\
(p $\leq$ 0.05).
\end{tabular}

timularon la firmeza del fruto, con valores estadísticamente similares a los valores que presentaron los biorreguladores AIA, AG y 6-BAP.

$\mathrm{El} \mathrm{pH}$ tuvo valores entre 4.5 y 4.7 , sin diferencias estadísticas entre tratamientos, pero los valores encontrados son similares a los que reportaron Casierra-Posada y Aguilar-Avendaño (2008). Sobre lo mismo Nuez (2001) indica que el jugo de tomate debe tener normalmente un $\mathrm{pH}$ de 4.0 a 4.5 para evitar el desarrollo de bacterias. Con respecto, al contenido de SST los valores oscilaron entre 4.4 y $4.6^{\circ}$ Brix, los cuales no son diferentes estadísticamente entre tratamientos, pero se encuentran entre los valores reportados de SST reportados para tomate (Crisanto et al. 2010). Sobre lo mismo Mejía et al. (2009), mencionan que el aumento de SST se debe a la hidrolisis del almidón al inicio de la maduración de frutos y la disminución de SST al aumentar de la respiración de los frutos maduros. Mientras que Vázquez et al. (2015) mencionan que el grado de SST para consumo en freso o para procesar debe ser al menos de $4.5^{\circ}$ Brix.

Las plantas de tomate del estudio, tuvieron un contenido de licopeno entre 18 y $21 \mathrm{mg} 100 \mathrm{~g}^{-1}$ de muestra fresca (Tabla 3), sin diferencias estadísticas entre tratamientos. Al respecto Shi y Le-Maguer (2000), reportan que los valores óptimos de licopeno son de alrededor de los $20 \mathrm{mg} 100 \mathrm{~g}^{-1}$ de muestra fresca. El contenido de vitamina $C$ fue mayor en los frutos de los extractos RT y RM, lo cual podría atribuirse a que los polifenoles son químicos bioactivos contribuyendo a la pigmentación de las plantas y a la actividad antioxidante (Stingu et al. 2012). Por lo que los extractos que tienen alto contenido de polifenoles como RT (Varela-Rodríguez et al. 2019) y RM (Jasso de Rodríguez et al. 2015), podrían promover la síntesis de vitamina $\mathrm{C}$.

\section{CONCLUSIONES}

Los extractos de RT, RM, FM, FR y CF, de plantas de las zonas semiáridas del noreste de México promueven el crecimiento, rendimiento y calidad de fruto en el cultivo de tomate saladette, con valores similares que el biorregulador 6-BAP. El extracto de $R$. muelleri mostró la mayor efectividad al promover el crecimiento y producción de fruto de tomate, por lo que representa una alternativa para la formulación de un bioestimulante de origen biológico que mejore la productividad de las plantas de tomate como consecuencia de los compuestos fenólicos y de la actividad sinergística entre la matriz de compuestos químicos que lo integran.

\section{AGRADECIMIENTOS}

A María Guadalupe Moreno Esquivel, Edith E. Chaires Colunga, Olga L. Solís Hernández, M. Leticia Rodríguez González y Juan Valenzuela Cabrera del Laboratorio de Fitoquímica de la Universidad Autónoma Agraria Antonio Narro, por su asistencia en la obtención de los extractos y su apoyo en las actividades de invernadero 


\section{LITERATURA CITADA}

AOAC (1990) Official Methods of Analysis. Association of Official Analytical Chemists. 15th Ed. Arlington, Virginia, USA. 384p.

Arias R, Lee TG, Logendra L, Janes H (2000) Correlation of lycopene measured by HPLC with the $L^{*}, a^{*}, b^{*}$ color readings of a hydroponic tomato and relationship of maturity with color and lycopene content. Journal Agricultural and Food Chemistry 48: 1697-1700.

Casierra-Posada F, Aguilar-Avendaño OA (2008) Calidad en frutos de tomate (Solanum lycopersicum L.) cosechados en diferentes estados de madurez. Agronomía Colombiana 26 : 300-307.

Crisanto A, Vera A, Chávez J, Carrillo J (2010) Calidad de frutos de tomates silvestres (Lycopersicon esculentum Var. Cerasiforme dunal) de Oaxaca, México. Revista Fitotecnia Mexicana 33 : 7-13.

FIRA (2017) Panorama Agroalimentario, tomate rojo 2017. Fideicomisos Instituidos en Relación con la Agricultura. México. 24p. https://www.fira.gob.mx/InfEspDtoXML/abrirArchivo.jsp?abreArc=65310. Fecha de consulta: 9 de mayo de 2019.

Giovannoni J (2001) Molecular biology of fruit maturation and ripening. Annual Review of Plant Physiology and Plant Molecular Biology 52: 725-749.

Gravel V, Antoun H, Tweddell RJ (2007) Growth stimulation and fruit yield improvement of greenhouse tomato plants by inoculation with Pseudomonas putida or Trichoderma atroviride: possible role of indole acetic acid (IAA). Soil Biology and Biochemistry 39 (8): 1968-1977.

Gutierrez T, Hoyos O, Páez M (2007) Ascorbic acid determination in Cape gooseberry (Physalis peruviana L.) for high-performance liquid chromatography (HPLC). Revista de la Facultad de Ciencias Agropecuarias 15: 70-79.

Ignat I, Stîngu A, Volf I, Popa VI (2009) Natural bioactive compounds as plant growth regulators. Scientific Papers, Series Agronomy 52: 187-192.

Ignat I, Stingu A, Volf I, Popa VI (2011) Characterization of grape seed aqueous extract and possible applications in biological systems. Cellulose Chemistry and Technology 45: 205-209

Ignat I, Radu DG, Volf I, Pag AI, Popa VI (2013) Antioxidant and antibacterial activities of some natural polyphenols. Cellulose Chemistry and Technology 47: 387-399.

Jasso de Rodríguez D, Trejo-González FA, Rodríguez-García R, Díaz-Jiménez MLV, Sáenz-Galindo A, HernándezCastillo FD, Villarreal-Quintanilla JA, Peña-Ramos FM (2015) Antifungal activity in vitro of Rhus muelleri against Fusarium oxysporum f. sp. lycopersici. Industrial Crops and Products 75: 150-158.

Jasso de Rodríguez D, Salas-Méndez EDJ, Rodríguez-García R, Hernández-Castillo FD, Díaz-Jiménez MLV, Sáenz-Galindo A, González-Morales S, Flores-López ML, Villarreal-Quintanilla JA, Peña-Ramos FM, CarrilloLomelí DA (2017) Antifungal activity in vitro of ethanol and aqueous extracts of leaves and branches of Flourensia spp. against postharvest fungi. Industrial Crops and Products 107: 499-508.

Mejía S, Vega M, Valverde J, López J, Caro J (2009) Effect of wax application on the quality, lycopene content and chilling injury of tomato fruit. Journal of Food Quality 32: 735-746.

Nuez V F (2001) El cultivo del tomate. Editorial Mundi- Prensa. Barcelona, España. 538p.

Parađiković N, Teklić T, Zeljković S, Lisjak M, Špoljarević M (2018) Biostimulants research in some horticultural plant species-A review. Food and Energy Security 8: 1-17. 
Pérez-Labrada F, Benavides-Mendoza A, Vázquez-Badillo ME, Ramírez H (2014) Addition of citric acid to nutrient solution of tomato cultivated in calcareous soil. Terra Latinoamericana 32: 251-255.

Ramírez H, Hoad GV, Benavides A, Rangel E (2001) Gibberellins in apple seeds and the transport of [ $\left.{ }^{3} H\right]$-GA4. Revista de la Sociedad Química de México 45: 47-50.

Ramírez H, Peralta-Manjarrez RM, Benavides-Mendoza A, Sánchez-López A, Robledo-Torres V, HernándezDávila J (2005) Efectos de prohexadiona-Ca en tomate y su relación con la variación de la concentración de giberelinas y citocininas. Revista Chapingo Serie Horticultura 11: 283-290.

Ramírez H, Zavala-Ramírez M, Sánchez-López A, Aguilar-Zárate P, Cristóbal-Aguilar N, Rodríguez-García R, López-Fabian A (2016) Tomato responses to bioregulators grown under greenhouse conditions. International Journal Plant Soil Science 10: 1-13.

SCFI (1998) NMX-FF-031-1997-SCFI. Productos alimenticios no industrializados para consumo humano. Hortalizas frescas. tomate - (Lycopersicun esculentum Mill.). Normas Mexicanas. Dirección General de Normas, SAGARPA. México. 15 p. https://www.colpos.mx/bancodenormas/nmexicanas/NMX-FF-031-1998.PDF. Fecha de consulta: 9 de mayo de 2019.

Shi J, Le-Maguer M (2000) Lycopene in tomatoes: chemical and physical properties affected by food processing. Critical Reviews in Food Science and Nutrition 40: 1-42.

Stevenson DG, Eller FJ, Wang L, Jane JL, Wang T, Inglett GE (2007) Oil and tocopherol content and composition of pumpkin seed oil in 12 cultivars. Journal of Agricultural and Food Chemistry 55: 4005-4013.

Stingu A, Volf I, Popa VI, Gostin I (2012) New approaches concerning the utilization of natural amendments in cadmium phytoremediation. Industrial Crops and Products 35: 53-60.

Talmaciu A, Tanase C, Volf I, Popa VI (2015) Influence of polyphenolic compounds on Ocimum basilicum L. development. Analele Stiintifice ale Universitatii “Alexandru Ioan Cuza”Din lasi. (Serie Noua). Sectiunea 2. a. Genetica si Biologie Moleculara 16: 83-88

Tanase C, Stingu A, Volf I, Popa VI (2011) The effect of spruce bark polyphenols extract in combination with deuterium depleted water (DDW) on Glycine max L. and Helianthus annuus L. development. Analele Stiintifice ale Universitatii "Alexandru loan Cuza"Din lasi. (Serie Noua). Sectiunea 2. a. Genetica si Biologie Moleculara 12: 115-120.

Tanase C, Vantu S, Popa VI (2013) "In vitro"effect of some industrial by-products on Lavandula angustifolia Mill. explant growth. Analele Stiintifice ale Universitatii "Alexandru Ioan Cuza"din lasi Sec. II a. Genetica si Biologie Moleculara 14: 13-18.

Tanase C, Talmaciu AI, Bâra IC, Boz I, Volf I, Oroian S, Popa VI (2018) New aspects of biomass waste valorization: spruce bark crude extracts as plant growth regulators. Bio Resources 13: 3994-4007.

Varela-Rodríguez L, Sánchez-Ramírez B, Rodríguez-Reyna IS, Ordaz-Ortiz JJ, Chávez-Flores D, Salas-Muñoz E, Osorio-Trujillo JC, Ramos-Martínez E, Talamás-Rohana P (2019) Biological and toxicological evaluation of Rhus trilobata Nutt. (Anacardiaceae) used traditionally in Mexico against cancer. BMC complementary and alternative medicine 19: 153.

Vázquez VP, García LMZ, Navarro CMC, García HD (2015) Efecto de la composta y té de composta en el crecimiento y producción de tomate (Lycopersicon esculentum Mill.) en invernadero. Revista Mexicana de Agronegocios XIX: 1351-1356. 